\title{
Hypermodels: An Exploration of New Media Environments and Expansive Representation of Architecture
}

\author{
SEHER ERDOĞAN FORD \\ Temple University
}

With the advent of new digital media technologies offering immersive virtual environments have emerged new modes of architectural representation. How, in turn, can these technologies shape the less visible, and visual, aspects of architectural production? This paper considers such digital, immersive technologies as Mixed, Virtual, and Augmented Reality within the historical and theoretical context of digital media to better understand their function in charting a frontier for the three dimensional representation of architecture. I propose the notion of the hypermodel, a "hypertext version" of digital models that contains and "opens up" to more than the physical parts of a building. Hypermodel is a connector of digital space and the physical worldrepresented via multiple forms of media-revealing the temporal expanse and informational depth of the virtual beyond the bounds of an architectural artifact. In this sense, the new medium also hints at and allows for novel collaborative methods. The new language of design and communication at work in the mixed reality medium is itself interconnectedit reflects and reinforces the inter-disciplinary and inter-media nature of architectural production today.

This paper establishes a conceptual framework and projects a methodology for future applications of such a hypermodel. To begin, it is helpful to better understand the context of and define "digital media" and "new media," as they are ubiquitous yet elusive terms. I will do this through a brief overview of the terms as employed in media studies and in contemporary discourse, and by identifying the most salient aspects that characterize the "new" in digital media. Then, I will discuss the relationship between new media and older media through the notion of "remediation" and, finally, explore new media's relevance to and adoption in architectural production. I will argue that digital 3D models endowed with new media capabilities, what I refer to as hypermodels, leverage the potential of the technology. By way of discussing several examples of recent collaborative applications of virtual environments in the building industry, I will follow with a closer reading of a digital reconstruction project of an architectural heritage site. The complexities of this and similar sites will exhibit the need for a hypermodel-a representational strategy that integrates immersive and heterogeneous media in relaying the tangible as well as the intangible aspects of architectural production.

\section{WHY BEGIN WITH MEDIA? (WHAT IS NEW MEDIA ANYWAY?)}

Broadly speaking, media refers to the means and channels of communication, and its genealogy includes oral histories, papyrus print, press print, and electronic dissemination in various forms. As an academic discipline, media studies has a relatively short but prolific past, with its scope and focus varied among distinct geographies. In the U.S., starting as early as the 1960s, media studies have resided primarily in mass communication departments and maintained close ties with film and TV production. In contemporary discourse, the term "new media" refers to the computerized means of communication and embraces anything within the digital realm, from mobile phones to virtual reality. The seminal digital media theorist Janet Murray explains the need for and the tendency toward such a broad sweeping and generic grouping. In her book, Inventing the Medium: Principles of Interaction Design as a Cultural Practice, Murray observes that unlike the previously established modes of media-such as books or newspapers-emerging media is still unstable in terms of its building blocks and design language. Advancements involve major innovation and invention as opposed to refinement. ${ }^{1}$ For this reason, the idea of the "new" still persists, and in fact, dominates the discourse. But Murray cautions against overusing the term "new media" because it puts more emphasis on its (technological) novelty than its (cultural) significance. To assess the medium as a function of a considered language of design, Murray proposes shedding the term "new" and keeping only "digital media." Since design is at the core of this paper, I will adopt this distinction as well.

Do virtual environments constitute their own medium? In an editorial in the journal Convergence, digital media theorists Maria Engberg and Jay D. Bolter introduce the questions around the emerging language of digital design and discuss the cultural implications influencing the production of virtual environments. ${ }^{2}$ They begin by explaining the origins of the virtual medium from the perspectives of art history, philosophy, and sociology. Perhaps most familiar to this paper's audience is the reference to the art critic Clement Greenberg's 
argument in the 1960s that every medium ought to develop its own unique characteristics. In this framework, Greenberg condenses his ideas into a call for "medium specificity." While artists and designers practicing within inter-media have since critiqued this inherently isolating view, its theoretical premise has remained influential. Engberg and Bolter, as an introduction to the issue entitled "Cultural Expression in in Augmented and Mixed Reality," draw parallels between Greenberg and the pioneering media theorist Marshall McLuhan, who broadened the definition of medium to include anything and everything that modulate our perception of time and space. In this paper, I argue that digital models as portals into virtual environments accomplish exactly that modulation of time and space but in such unique ways that they should be considered their own evolving medium.

However rapidly proliferating, the developments in digital media make up a continuum. Bolter identifies in several of his works the generative step from preceding forms to the newer version of a medium $\neg-$ he terms this transformation an act of "remediation." By his definition, remediation is the process in which a new form replaces the older one by adopting some of its features, modifying others, and ultimately reshaping its presence within the cultural context. ${ }^{3}$ For example, Bolter discusses how writing on papyrus remediated the orally disseminated word, and how hypertext remediated text by mimicking the older form visually while serving as a departure point for other intertextual content. In "Spatialization: A Strategy for Reading Narrative," Susan Stanford Friedman describes textual narrative's relationship to spatial domains even more precisely. Narrative represents space in two axes-vertical and horizontal-where both allow movement through space and time in different ways. ${ }^{4}$ The horizontality refers to the sequence of ideas in one context and the verticality implies a sense of going deeper into one idea and its associations with others in different contexts. Hypertext exists at the intersection of these two axes as an access point to both spatial and temporal navigation.

\section{"REMEDIATION" OF ARCHITECTURAL REPRESENTATION: HYPERMODELS}

With digital media now such an integral part of its production and representation, how can architecture be "remediated" toward more interactive forms? Sylvia Lavin, in "Architecture Animé or Medium Specificity in a Post-medium World," offers a historical and geographic account of architecture's search for its own possibility as a dynamic medium, since the 1960s and across the US, Italy, and Japan. ${ }^{5}$ Soon after Greenberg's calls for medium specificity, the art and design world began to shed single-medium defined practices and to seek crossdisciplinary modes of expression. Influenced by the work of artists such as Andy Warhol, many architects' visions for the cross-medium and post-medium took the form of participatory and multisensory experiences, presented primarily as a critique of the immutability or the "frozen" nature of architecture. As an example, Lavin describes a project by an Italian architecture student who superimposed orthographic drawings on mylar onto neon lights to create spatial effects mimicking axonometric drawings. Lavin likens this "apparition" to how digital 3D models work today. Even though the results of these media boundary-exploring works predominantly created a disorienting environment of fragmentary and confusing space, Lavin contends that architecture, as a physical endeavor, will prevail, albeit while transforming into a "container" for digital media.

While Lavin's focus is on the relationship of the built work to digitized data, it is also about the representation of the physical artifact in digital space. The neon supported hologram drawings, which for her signaled precursors to 3D digital models, are an example of "legacy formats" holding on to obsolete aspects of an earlier technology at the expense of curtailing its remediation. I will argue that the power of the digital model is less to do with the transformation from $2 D$ to $3 \mathrm{D}$ or even to building information modeling (BIM) platforms and more to do with how a "hyper" spatial domain emerges out of the representation of the architectural construct. This domain includes multimedia elements such as wikis, geospatial maps, and videos, and links to content from diverse disciplines, which are currently not typically supported by architectural representation technologies. It also shifts the perspective of the "user" to one of "interactor" (a term Murray strongly promotes), who engages with the digital space in real-time. Hence, I propose the term hypermodel as a remediation of the 3D digital platform and facilitator of a new, unique medium in two ways. One, it contains the information and interactive design language to represent the building changing over time. Two, it situates the architectural project in its sociocultural context through connections with heterogenous data. To use Stanford Friedman's analogy, hypermodels exist at the intersection of the horizontal (temporal) axis and the vertical (contextual) axis. It achieves both of these by facilitating an immersive environment, in which the interactor occupies both the new digital and the physical spatial domain.

More than simply a self-contained digital 3D model, the hypermodel gives access to a "hyper" environment endowed with a suite of possible applications. Current modes of this environment include what are commonly termed Virtual Reality (VR), Augmented Reality (AR), and Mixed Reality (MR). VR produces a digital representation of a space where the interactor might navigate freely, and engage with other participants and the design interface to extract more information or adapt the settings. VR is an entirely immersive experience, where the interactor is willingly "fooled" into believing that they are present at a place other than the one they physically occupy. While in VR the digital space of the HyperModel is dominant and seemingly all-encompassing, AR relies on layering. The digital is layered on top of the physical to create access points into the virtual world through specific trajectories that enhance the experience of the physical world. In AR, the hypermodel is in the background, serving the interactor immediately and in seemingly invisible ways. Lastly, MR operates on a robust reciprocity between the digital representational space and the physical one, where objects in either space can interact with each other in real-time. If hypermodels generate a spectrum of relationships between the digital and physical, thereby a range of experiences for the interactor, MR occupies the middle where there is complete overlap and possible negation of the distinction between 
the real and the virtual. The spaces are entirely fluid, extending into each other's domain and mixing with one another.

I have proposed that the hypermodel is the remediated form of 3D digital models. Next, I want to look more specifically at how the new medium facilitates architectural representation and what kind of messages it generates. To do that, I want to briefly consider the idea of "virtuality" and reference the philosopher Elisabeth Grosz via her collection of essays entitled Architecture from the Outside. ${ }^{6}$ Grosz contemplates virtuality and its history as part of the cognitive process. The human mind entered virtual space as early as the wall paintings of the caves in Lascaux, France, and mastered the craft of virtual worlds through the perspectival construction of Renaissance paintings. In another sense, narrative fiction pulls one into a virtual space. However immersive it is, Grosz argues, the virtual world is not an alternative but an extension of the real world. Virtual adds, shifts, enhances, but cannot replace. In that sense, Grosz asserts the separation of virtual from simulation.

This distinction is powerful for architectural representation because it frees the 3D representation project from one that is bound by the pursuit of photorealism-singular likeness-and allows for the pursuit of expression and synthesis-generative multiplicity. Hypermodels are able to incorporate varied content beyond and underlying the building as a physical artifact. Interdisciplinary scholarship and research find a spatial container in the hypermodel and reveal the intangible factors pertinent to an architectural project. Due to their interdisciplinary and inter-media nature, hypermodels are projects of ongoing collaboration. As a tool, the hypermodel generates a new medium of design, delivery, and operation, and, in turn, the medium alters the culture of work within the industry. The following examples show how the hyper virtual environments, enabled by various technologies, serve the architectural project as a collaborative endeavor.

\section{COLLABORATION THROUGH HYPERMODELS}

As an alternative to studying collaboration between specific stakeholders in the design process-whether from the industry or academia-I propose to trace the outcomes of the medium itself. In other words, my selection of the following projects was based on the fact that they implemented hypermodels, rather than on the roles of those involved. Through a closer look at the interdependency between the digital and physical spaces involved in each project, I argue that new medium of the hypermodel both requires and alters collaboration.

The first example aims to better integrate the domains of the "designer" and "builder." The "designer" typically includes architects and consulting engineers and other kinds of designers; builders include the construction manager or the general contractor and subcontractors, and the project management team. As part of the 2016 AEC Hackathon in Helsinki, Finland, one of the hosts, SWECO, a Finnish construction and engineering company challenged the participating teams to find technological solutions to making the shared work between project stakeholders more collaborative.? "Team Safety," whose members included technologists from TrimbleConnect, the cloud-based collaboration platform designed to integrate with BIM and other software, decided to propose a solution to improve communication between the field and the office, with the goal of increasing job safety.

The proposed scenario is a remediation of the BIM model, in that it implements AR technology to bridge digital and physical space at full scale. This bridge is bidirectional, placing the designers on the job site through digital space and allowing the builder access to the digital representation of the project in real time. The digital model becomes a dynamic reference during the construction process. This example works to improve existing models of collaboration between industry partners by facilitating a shared, dynamic platform and realtime data input. As a result, the data lost in translation is minimized

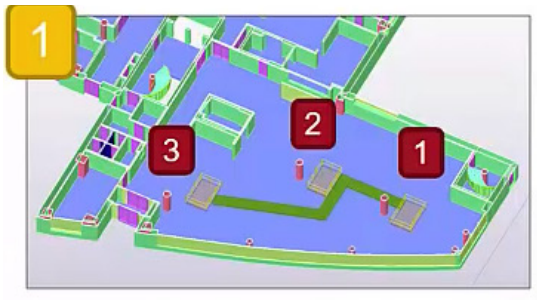

Plan in Office
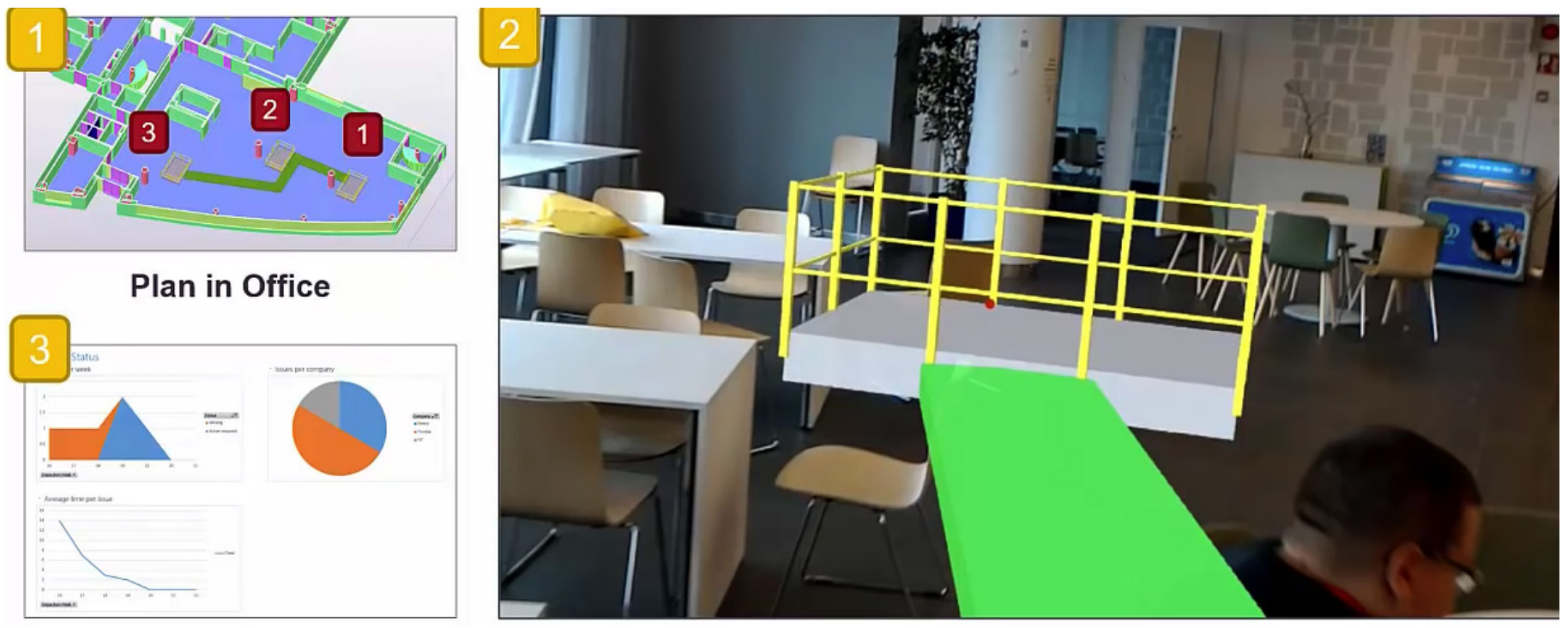

Figure 1: Screen capture of "Team Safety" presentation 


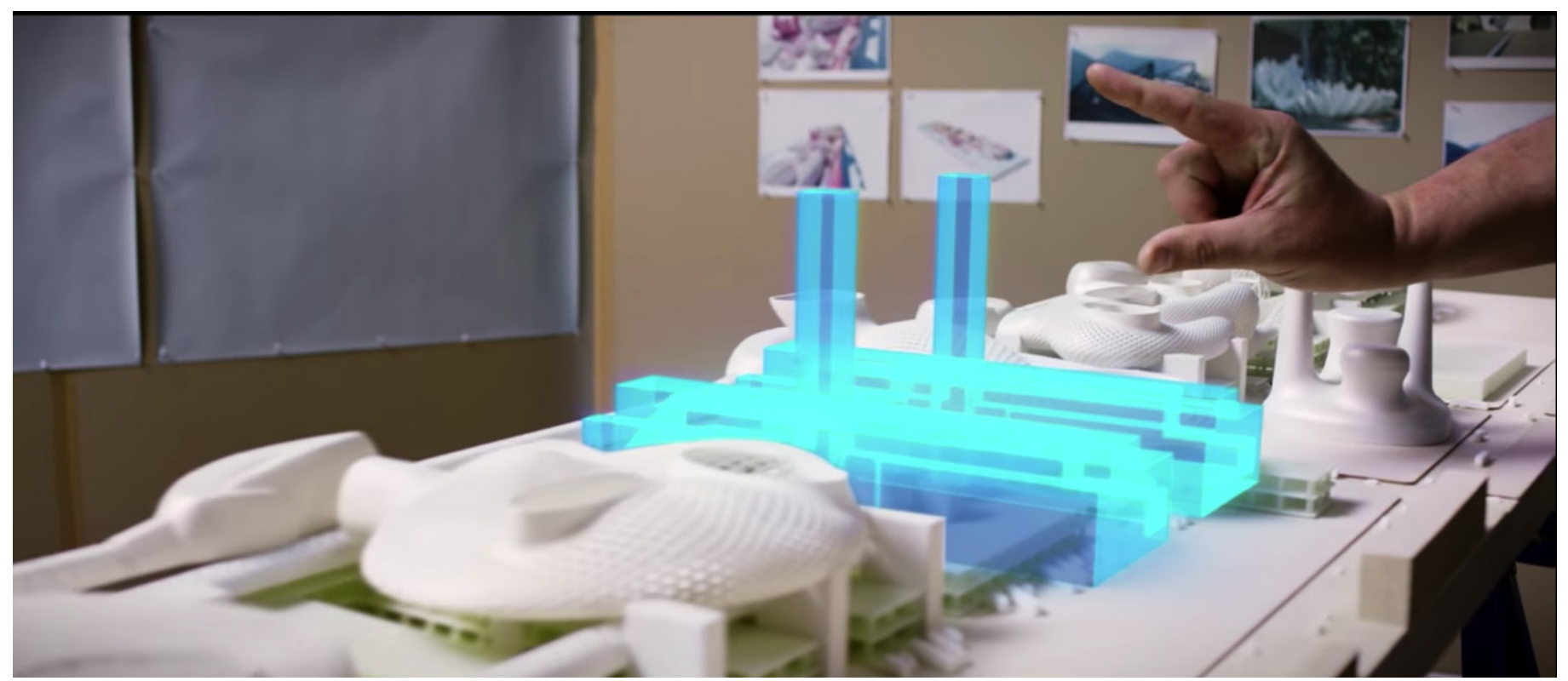

Figure 2: Screen capture of Lynn and HoloLens partnership video

and information "lives" not only within the digital model but also in the extension of the digital model located in the physical space. Annotation is contextualized and obsolescence eliminated.

While job site safety is the "test case" for this project, the technology can be applied to other critical aspects of construction, an effort that requires dynamic and synchronized communication between the various parties involved. Its facility to enhance communication can also aid post-construction activities related to the operation of the building, such as commissioning, post-occupancy data analysis and maintenance. In this sense, processes of design, building, and operation can use the same hypermodel, which remains current throughout the life cycle of the building and serves short and longterm problems demanding spatial coordination among team members with different areas of expertise. The "bridge" between the office and field can be multi directional and engage with other routes of communication.

The second hypermodel project is a collaboration between the architecture office Greg Lynn FORM, and Microsoft in partnership with Trimble in the development of the wearable AR device HoloLens. ${ }^{8}$ The context for the project is the 2016 Venice Architecture Biennale. The organizers invited Lynn to work on a re-use design project of the Packard Plant in Detroit, the abandoned site of a former automobile factory. In a video promoting the partnership and Lynn's initial use of the AR headset, the architect describes the details of the project brief and the early phases of design. Standing next to a physical site model of the super-block and wearing the headset, Lynn enters into the digital space of the hypermodel (the video switches to his point of view). To better grasp the scale of the Packard Plant site, he accesses the digital library provided through TrimbleConnect and "grabs" with his hand a model of the Tate Modern in London. An iconic building well-known to him and other designers, the Tate serves as a volumetric reference. By replicating the digital massing model layered on the physical site model, Lynn concludes that his project is equal to twelve Tate Moderns.

In this mode, the architect is collaborating in a general sense through the digital libraries with the creators of the data stored there. The digital library becomes the agent of collaboration while the act of working together happens primarily extemporaneously. The shared activity proliferates to other times and places, where the designer has a team of distant partners contributing to the work in various ways. Architects' primary role in collaborative work is most often the task of synthesis. This process of absorbing, processing, and editing heterogeneous data is better supported through AR technology because it enables not only faster but more educated decisionmaking. In terms of the affordances of hypermodels, the designer can go deeper into the "vertical" space of the model-the cultural and sociocultural data-to access the history of the neighborhood, for example, and inform decisions regarding say program and use. The hypermodel negates the illusion of the architect creating in isolation and reinforces the networked nature of digital space, containing the input of a diverse set of contributors, where all critical decisions are made.

The video of Lynn utilizing the AR headset demonstrates a physical representation-in this case a massing model-functioning as an anchor onto which electronic media can be tethered. The Trimble library, as the container of a networked and scaleless database, exists in the digital realm but finds context within the physical representation to a particular scale. It exemplifies augmented reality, in which digital layers reveal or enhance an aspect of the physical "base." Lynn uses the hypermodel to cross-reference scalar or formal information, however, one can speculate that the comparison does not need to be only in terms of volume but can draw corollaries between commercial, historical, social, and logistical databanks. Given the history of the site, the references informing the design could conceivably come from a 
multitude of sources related to the heritage of the automotive industry in the city.

The last project example involves new media technologies as implemented in the digitization of cultural heritage sites. Initiated in 2015, it marked a collaboration between the UNESCO Chair in "Management and Promotion of World Heritage Sites: New Media and Community Involvement," at Kadir Has University, Istanbul, Turkey, and the leading Turkish BIM software distributor, Bilkom. The team consisted of myself, other faculty members Assoc.Prof. Yonca Kosebay Erkan and Prof. Füsun Alioglu; undergraduate students from the Architecture department at Kadir Has, and technical experts on $A R C H I C A D$, the building information modeling software represented by Bilkom in Turkey. Therefore, one educational goal of the project was for the students to learn and practice the collaborative use of this specific platform.

The team selected the Studius Monastery Church, later known as the Mosque of Imrahor, as an appropriate initial case study for a digital reconstruction project. Built near the Golden Gates of Constantinople in the 5th century, the Studius Monastery was not only a religious destination but also a center of cultural and intellectual life in Byzantine Empire. The oldest surviving religious building in the city, the site has been abandoned for decades, but is slated for a controversial and imminent renovation to convert it back to an operational mosque. Over the course of 16 centuries, due to changes in ownership and use, numerous powerful earthquakes and fires, and other beautification projects, the church went through a series of architectural modifications -in the form of fills, extractions, and overlays - at varying scales and scope, resulting in a build-up of material layers, albeit with little legibility in terms of their provenance. The resulting current physical artifact is an amalgamation of its layers of reconstruction, making its translation to BIM extremely challenging.

Along with digital modeling, the team also engaged in close analysis of existing documentation of the building researched the historical "layers" of the architectural and geographic site. The technological challenges necessitated a phased approach to modeling, in which the initial installment of the project focused on a single aspect of the site's history. The team decided to transfer the Byzantine basilica in its 11th century condition, at the peak of its social prominence and architectural presence, to the digital environment of ARCHICAD. This decision entailed the task of deciphering and uncovering later modifications. The relatively narrow focus was motivated by the academic calendar of the university as well as the steep learning curve the software initially demanded.

The technical challenges of collaborating upon the digital reconstruction of a historic building created a critical limitation, reducing the physical, temporal, and inevitably, conceptual scope of the project. The major challenge with ARCHICAD was the question of how to represent or recreate the Roman masonry structure along with the intricate finish work within the interior, all while implementing a tool specifically tailored to contemporary standards and components of construction. Therefore, a "well-built and clean" model and a "finished" visualization of the basilica would essentially erase the sense of time-and represent

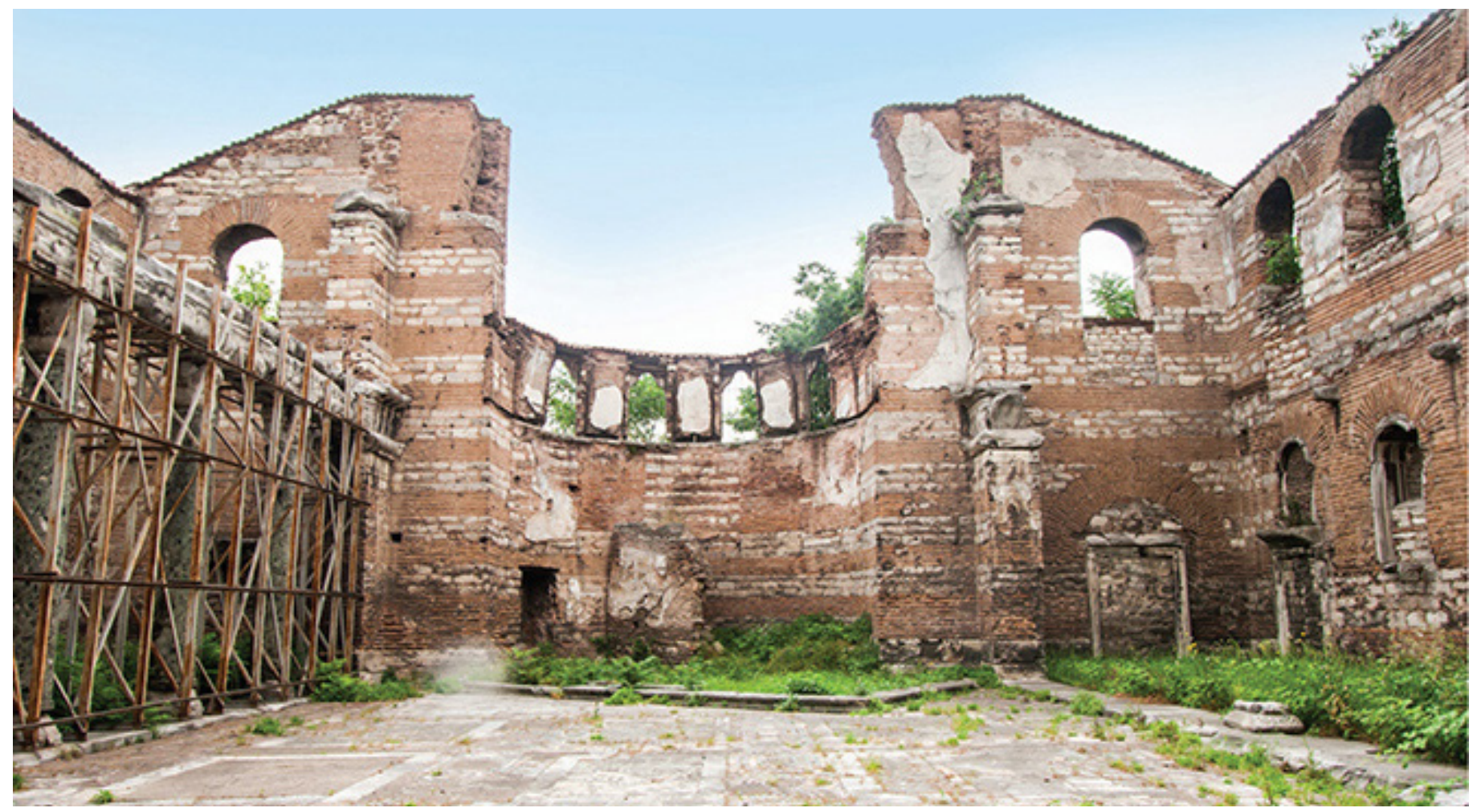

Figure 3: View of the current state of the apse. (Photograph by Esra Kudde) 


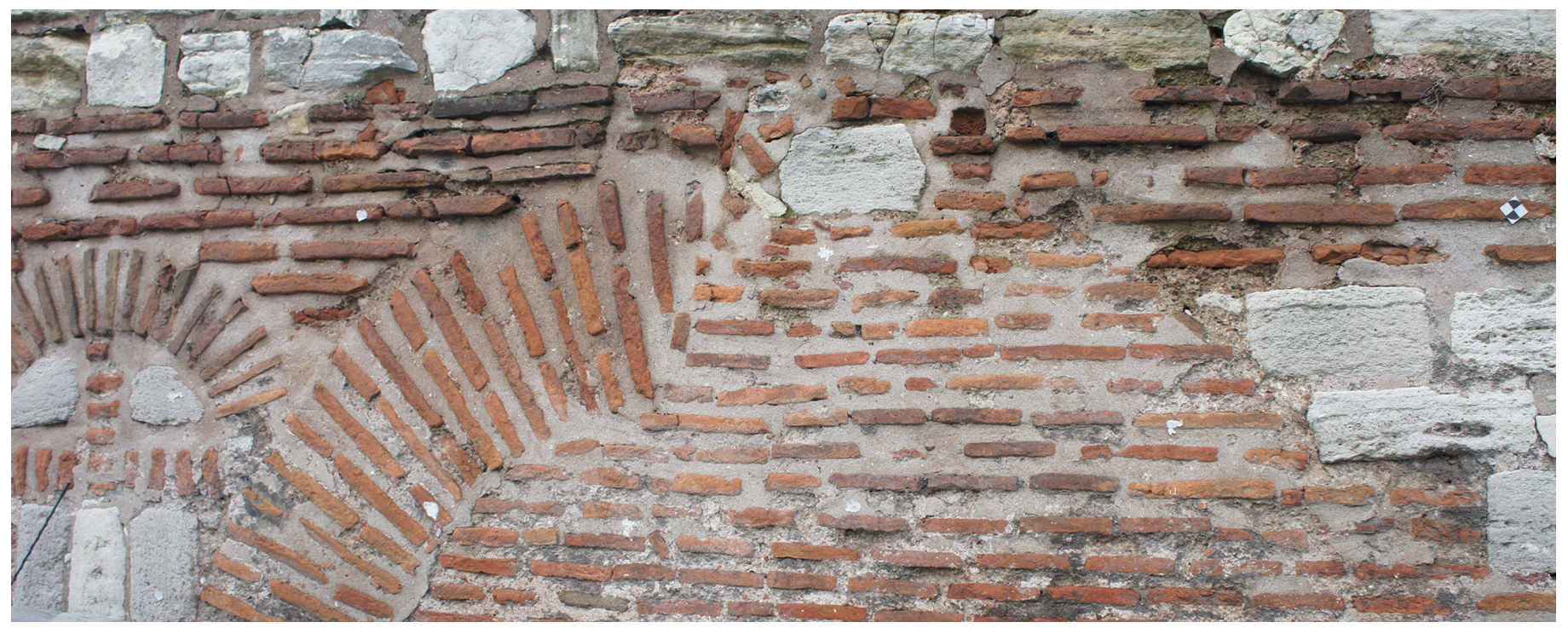

Figure 4: Exterior wall detail (Photograph by the author)

a singular, arguably less accurate, rendition of history. Furthermore, a robust BIM platform would necessarily encapsulate the project within a specific temporal identity and a particular set of industry standards. Instead of a purely logistical BIM model, the representation of such a site would require essentially a "sociocultural BIM" model.

How could the implementation of a hypermodel improve future phases of this project? The speculative answer revisits Stanford Friedman's analogy of horizontal and vertical axes in digital space, and shows how it becomes critical in conceptually organizing information on the site. The hypermodel allows the interactor to navigate "horizontally" within the representational space-between multiple instances along the timeline of the building - thereby achieving a synthetic understanding of its continuous material transformation through the course of centuries. One can stand in the 5th century basilica and juxtapose it against the architectural modifications carried out during its conversion to a mosque. The immersive experience powered by hypermodels in MR mode removes the conceptual "edge" around the digital model such that the interactor is present within the digital space, where layers of intangible "drivers" behind each instance of reconstruction are revealed. For instance, the change in Islamic liturgical practice that required more private interior spaces show up in the digital model as a series of masonry fills completed in the 17th century. The hypermodel also allows the interactor to navigate "vertically" between multiple object scales, honing in on the full extent of its construction and its basis in traditions of fabrication, networks of material sourcing, and historically expressive motifs. Akin to the familiar action of visually "zooming in and out" in digital space, the interactor occupying the hypermodel "zooms in" to investigate the details of the chemical composition of assembly components and "zooms out" to see building scale finish patterns. Furthermore, MR mode opens the model up to other data libraries such that, when standing at the apse of the Church of Studius and studying the stone paneling on the interior walls, one can "pull up" the visual documentation on other Byzantine monuments and compare their designs or access literature on Eastern Roman visual culture to analyze details of stone carvings. While the hypermodel's full capabilities are still speculative, my ongoing research deals with the integration of the archival data on the building within a dynamic 3D model presented in an interactive, web-based and virtual reality environment.

\section{CONCLUSION}

Architects must consider digital models in the context of media and new capabilities not simply as a robust platform for storing and sharing information regarding the physical elements of a building, but as a dynamic and interactive domain of communication and medium of representation that reveals the intangible aspects of architectural production. These so-called hypermodels, akin to the functionality of hypertexts, both contain and connect to outside sources of information. Embedded within them are multiple scales of architectural information as they change along the temporal axis. As a connector to other types of information, the hypermodel provides access to a variety of digital libraries, which in turn updates the model with heterogeneous and dynamic data. The hypermodel is intrinsically an interdisciplinary endeavor. However, it goes beyond bidirectional sharing of information and spatializes the exchange of information. More so, the hypermodel contextualizes the larger project relative to its social, cultural, and political contingencies. This renewed perspective reinforces the medium of collaboration within the professional and academic field while also expanding it past the common disciplinary delineations. The collaborative architectural endeavor promises more than the efficient multiplication of team players: as architects, we must envision the critical proliferation of access to an expansive breadth and immersive depth of knowledge.

\section{ENDNOTES}

1. Janet H. Murray, Inventing the Medium: Principles of Interaction Design as a Cultural Practice, (Cambridge, Mass.: MIT Press, 2012), 8.

2. Jay David Bolter and Maria Engberg, "Cultural Expression in Augmented and Mixed Reality," Convergence Vol. 20 (2014): 4.

3. Jay David Bolter, The Writing Space: The computer, Hypertext, and the History of 
Writing, (Hillsdale, NJ : L. Erlbaum Associates, 1991).

4. Susan Stanford Friedman, "Spatialization: A Strategy for Reading Narrative," Narrative Vol. 1 14, No. 1 (1993): 14.

5. Sylvia Lavin, "Architecture Animé or Medium Specificity in Post-Medium World," (paper presented at Die Realitat des Imaginaren Architecktur und das digitale Bild, 10 Internationales Bauhaus-Kolloquium, Bauhaus, Germany, April 2007).

6. Elizabeth Grosz, Architecture from the Outside: Essays on Virtual and Real Space, (Cambridge, Mass. : MIT Press, 75-91.

7. “TrimbleConnect's Team Safety," AEC Hackathon, accessed January 10, 2017, https://www.youtube.com/watch?v=qazLCXVndhk.

8. 09_Endnotes. Clita accusata corrumpit eos at, animal imperdiet ei vim. Bonorum volumus nostrum eam ne, est erat tempor dissentiunt eu. Eu usu.

9. "Microsoft Hololens: Patner Spotlight Greg Lynn," Microsoft Hololens, accessed January 10, 2017, https://www.youtube.com/watch?v=1gmkJqWOWFQ. 